\title{
Density Functional Theory Calculation of Band Gap of Iron (II) Disulfide and Tellurium
}

\author{
Arumona A. E., Amah A. N. \\ Department of Physics, University of Agriculture, Makurdi, Benue. Nigeria
}

dai: https://doi.org/10.21467/ajgr.3.1.41-46

*Corresponding Author email: ajax5use@gmail.com

Article History

Received: 26 October 2017

Revised: 15 November 2017

Accepted: 17 November 2017

Published: 18 November 2017

Student(s)

- $\quad$ Arumona A. E.

Academic Year: 2016-2017

Course Level: Master Degree

Course Name: M. Sc. (Theoretical physics)

Course year: $2^{\text {nd }}$ Year

Mentor(s)

- Amah A. N.

\begin{abstract}
In this work, the band gap of Iron(II) disulfide and Tellurium were computed using the density functional theory with different metaGGA functionals. The results showed that the meta-GGA functional M06L gave the closest value of the band gap as $0.96 \mathrm{eV}$ compared to the experimental value of $0.95 \mathrm{eV}$ for Iron(II) disulfide while the meta-GGA functional BJ06 gave the closest value of the band gap as $0.339 \mathrm{eV}$ compared to the experimental value of 0.335 $\mathrm{eV}$ for Tellurium. This study showed that the meta-GGA functionals M06L and BJ06 can effectively predict the band gap of Iron(II)disulfide and Tellurium. In general, the use of meta-GGA functionals can be extended to compute the band gap of other semiconductors.
\end{abstract}

Keywords: Iron (II) disulfide; Tellurium; Band gap; Density functional theory; meta-GGA functional; Semiconductor

\section{Introduction}

Iron (II) disulfide $\left(\mathrm{FeS}_{2}\right)$ is a semiconductor which belongs to the space group $T_{h}^{6}-\mathrm{Pa} \overline{3}$. It has a cubic crystal system (primitive cubic). Iron (II) disulfide is used in Lithium metal sulfide batteries, solar photovoltaics, battery recycling and molding compounds. It has a narrow band gap value of $0.95 \mathrm{eV}$ [1]. Tellurium (Te) is classified as metalloid (p-type semiconductor) and belongs to the group 16 in the periodic table known as the oxygen family. Tellurium belongs to the space group of $\mathrm{P}_{31} 21$ and its crystal atoms are two folds. Tellurium is used in the steel industry and as a thermoelectric device [2-3]. It is also used in solar cells in form of Cadmium Telluride $(\mathrm{CdTe})$. Tellurium has shown great promise in solar cell electric power generators and as a result production of Tellurium is in high demand.

Band gap is the range of energy in a solid where there is no existence of electron states. It is the most basic property of a solid. It is a very important property which is used in the design of semiconductor devices and is also used to determine the electrical and optical properties of materials [4]. A known fact is that density

Copyright (C) 2017. The Author(s). Published by AIJR Publisher (India).

This is an open access article under Creative Commons Attribution-NonCommercial 4.0 International (CC BY-NC 4.0) end user license. 
functional theory is not very successful in predicting band gap of semiconductors. The prediction of energy band gap of semiconductors by density functional theory has been center of discussion over the years and still an active research area. The hybrid and meta-GGA functionals over the years have been developed. Vijay et al., [5] using density functional theory with TB-mBJ potential calculated the band gap of Iron (II) disulfide to be $1.186 \mathrm{eV}$. Quin et al., [6] using density functional theory with local density approximation (LDA) calculated the band gap of Iron (II) disulfide to be $0.6 \mathrm{eV}$. Zhang et al. [7], using density functional theory with GGA+U, GGA (generalized gradient approximation) and HSEO6 calculated the band gap of Iron (II) disulfide to be $0.81 \mathrm{eV}, 0.50 \mathrm{eV}$ and $2.66 \mathrm{eV}$ respectively. Tellurium band structure was calculated by Junginger [8] using a modified APW method. The band gap of $0.45 \mathrm{eV}$ at zero temperature was obtained compared to experimental value of $0.335 \mathrm{eV}$.

In this work, the band gap of Iron (II) disulfide and Tellurium were computed by using different meta-GGA functionals within the framework of the density functional theory. To the best of my knowledge there is no study that have computed the band gap of Iron (II) disulfide and Tellurium using different meta-GGA functionals within the density functional theory. A meta-GGA functional uses the kinetic density of the second order derivative with the magnitude and density of the gradient of the density. The meta-GGA functional are dependent on both kinetic energy density and Laplacian of the density [9].

\section{Computational Method}

The ABINIT code was used in the computation, this code is based on plane waves and pseudopotentials. The Hartwigsen-Goedecker Hutter pseudopotentials and different types of meta-GGA functional were used in the ab initio calculations [10]. Kohn-Sham method is applied in density functional theory and estimates the exact energy and density of many body electrons. Density functional theory calculates ground state properties and these calculations are done through the approximation exchange correlation potential [11]. For the purpose of this work, the number of bands used were 15, 20, 25 and 30. The k-points used to sampled the Brillouin zone were 70,120 and 180. The Cif2cell program was used to generate the geometrical setup for Iron (II) disulfide and Tellurium [12]. The Iron (II) disulfide and Tellurium CIF (Crystallographic Information Framework) files were obtained from crystallographic open database [13-14].

\section{Theory}

The density functional theory developed by Kohn-Sham is termed the modern density functional theory and is widely used across many fields.

The Kohn-Sham equation which resembles Schrodinger equation is given as:

$$
\begin{aligned}
& {\left[-\frac{1}{2} \nabla^{2}+V_{\text {eff }}^{\sigma}\right] \psi_{i}^{\sigma}(r)=\varepsilon_{i}^{\sigma} \psi_{i}^{\sigma}(r)} \\
& V_{e f f}^{\sigma}=V_{e f f}(r)+V_{H \text { artree }}[n]+V_{x c}^{\sigma}\left[n^{\uparrow}, n^{\downarrow}\right] \\
& n^{\sigma}(r)=\sum_{i} f_{i}^{\sigma}\left|\psi_{i}^{\sigma}(r)\right|^{2}
\end{aligned}
$$

where $V_{\text {eff }}^{\sigma}$ is the effective potential of spin $\sigma\left(V_{\text {eff }}, V_{\text {Hartree }}\right.$ and $V_{x c}^{\sigma}$ are the potential, Hartree potential and the exchange correlation potential of $\operatorname{spin} \sigma$ ) for a system of $n=n^{\uparrow}(r)+n^{\downarrow}(r)$ having independent electrons at

point $\mathrm{r}$, the ground state has one electron in each of the $N^{\sigma}$ orbitals $\psi_{i}^{\sigma}(\mathrm{r})$ with the lowest eigenvalues $\varepsilon_{i}^{\sigma}$. Equation (3) is the electron density of spin $\sigma$. The solution of the Kohn-Sham self-consistent equations is 
represented in a flow chart as shown in Figure 1. The energy exchange correlation of the meta-GGA functional is given as:

$$
E_{x c}\left[n_{\uparrow}, n_{\downarrow}\right]=\int d^{3} r e_{x c}\left[n_{\uparrow}(r), n_{\downarrow}(r), \nabla n_{\uparrow}(r), \nabla n_{\downarrow}(r), \tau_{\uparrow}(r), \tau_{\downarrow}(r)\right]
$$

where $e_{x c}$ is the exchange correlation energy density

$$
\begin{aligned}
& n_{\sigma}(r)=\frac{1}{\Omega_{B Z}} \sum_{i} \int_{B Z} d^{3} k \theta_{i \sigma}(k)\left|\psi_{i k \sigma}(r)\right|^{2} \\
& \tau_{\sigma}(r)=\frac{1}{2 \Omega_{B Z}} \sum_{i} \int_{B Z} d^{3} k \theta_{i \sigma}(k)\left|\nabla \psi_{i k \sigma}(r)\right|^{2}
\end{aligned}
$$

equations (5 and 6) are the spin density and kinetic energy density of spin $\sigma$. Where $\Omega_{B Z}$ is the first Brillouin zone volume, $i_{\text {is the band gap index, }} \theta_{i \sigma}=\theta\left[E_{F}-\in_{i k \sigma}\right]_{\text {is the heavy-side step function ( }} E_{F}$ is the Fermi energy and $\in_{i k \sigma}$ is the Kohn-Sham orbital energy) and $\psi_{i k \sigma}$ is the Kohn-Sham orbital normalized in one unit cell [15].

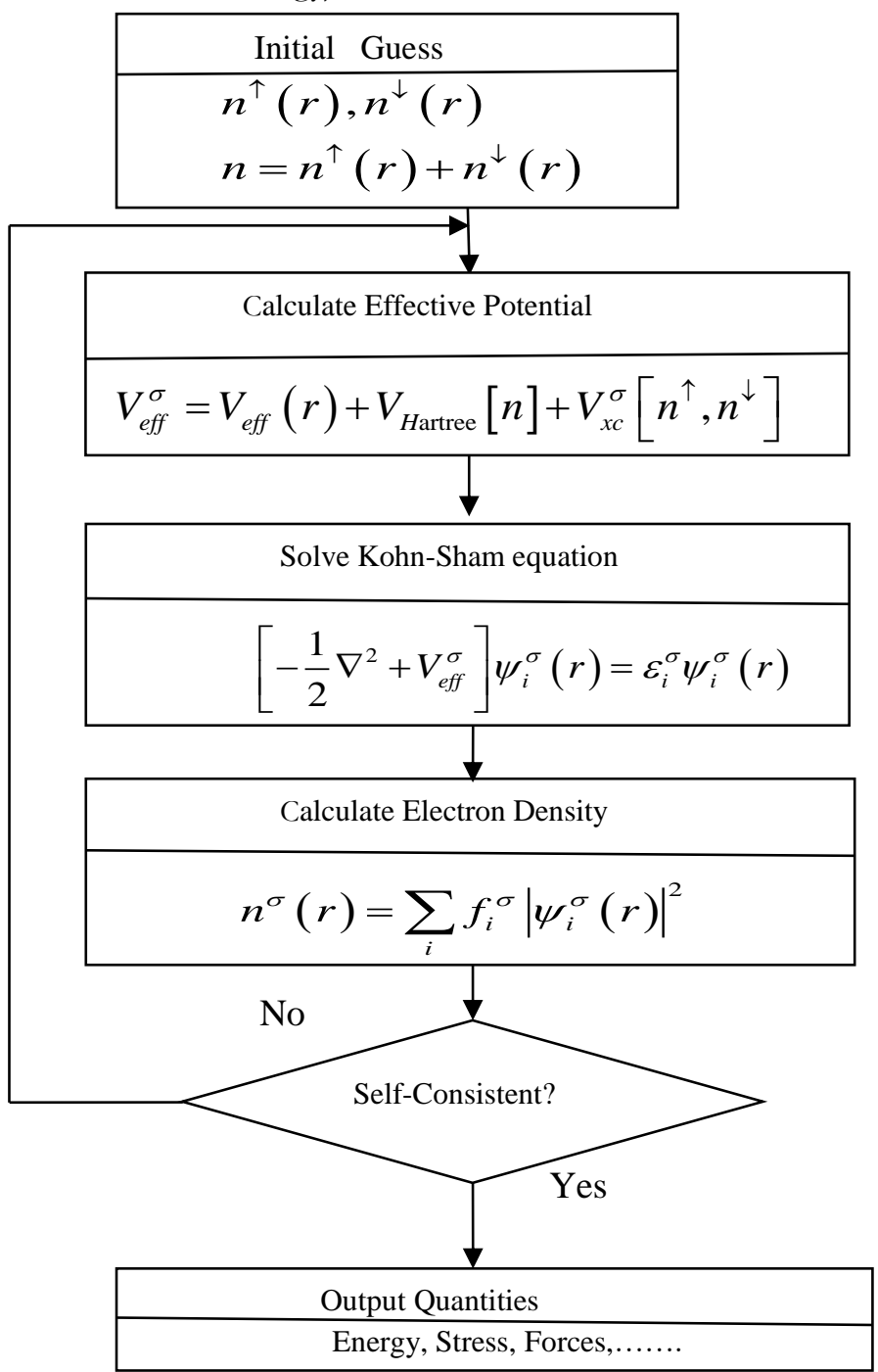

Figure 1: Representation of the Loop for Solution of Kohn-Sham Equation [11]

In general, the iteration of the two loops are done simultaneously for the two spins. The spins potential is a functional of the density of the spins. 
Density Functional Theory Calculation of Band Gap of Iron (II) Disulfide and Tellurium

$4 \quad$ Results and Discussion

The meta-GGA functionals that were used in this work are given as follows: XC_MGGA_X_LTA[16], XC_MGGA_X_TPSS[17],XC_MGGA_X_M06L[18],XC_MGGA_X_GVT4[19],XC_MGGA_X_TAU_HC TH[20],XC_MGGA_X_BR89[21],XC_MGGA_X_BJ06[22],XC_MGGA_X_TB09[23],XC_MGGA_X_RPP 09[24], XC_MGGA_C_TPSS[17], XC_MGGA_C_VSXC[19]. For Iron (II) disfulside 15 and 20 number of bands predicted it to be a metal with no band gap value.

The meta gga functionals (ixc $=-206$ to -232 ) gave band gap values for Iron (II)disulfide that are not very close to the experimental value and their band gap values for both 25 and 30 number of bands all converged to the same value at $120 \mathrm{k}$-points. The meta gga functionals (ixc $=-201$ to -205) gave band gap values for Iron (II) disulfide that are very close to the experimental value and their band gap values converged at $180 \mathrm{k}$-points, where meta gga functionals (ixc $=-203$ to -205 ) band gap values for both 25 and 30 number of bands all converged to the same value at $180 \mathrm{k}$-points. The meta gga functional (ixc $=-203$ ) gave band gap value for Iron (II) disulfide that is closest to the experimental value. For the meta gga functionals (ixc =-201 and -202) band gap values for Iron (II) disulfide for both 25 and 30 number of bands all converged to different values $(0.62 \mathrm{eV}, 0.58 \mathrm{eV}$ and $0.98 \mathrm{eV}, 0.91 \mathrm{eV}$ respectively) at $180 \mathrm{k}$-points.

Table 1: The computed band gap ( $\left.E_{g}(\mathrm{eV})\right)$ of Iron (II) disulfide with the meta-GGA functionals

\begin{tabular}{|l|l|l|l|}
\hline ABINIT code & Exp. $\left(\mathbf{E}_{\mathbf{g}}(\mathbf{e V})\right)[\mathbf{1}]$ & $\mathbf{E}_{\mathbf{g}}(\mathbf{e V})$ & Functional \\
\hline ixc $=-201$ & 0.95 & 0.62 & \\
ixc $=-202$ & 0.95 & 0.98 & LTA \\
ixc $=-203$ & 0.95 & 0.96 & TPSS \\
ixc $=-204$ & 0.95 & 1.03 & M06L \\
ixc $=-205$ & 0.95 & 1.10 & GVT4 \\
ixc $=-206$ & 0.95 & 0.77 & TAU_HCTH \\
ixc $=-207$ & 0.95 & 0.66 & BR89 \\
ixc $=-208$ & 0.95 & 0.55 & BJ06 \\
ixc $=-209$ & 0.95 & 0.82 & TB09 \\
ixc $=-231$ & 0.95 & 0.23 & RPP09 \\
ixc $=-232$ & 0.95 & 0.72 & TPSS \\
\hline
\end{tabular}

Table 2: The computed band gap $\left(E_{g}(\mathrm{eV})\right)$ of Tellurium with the meta-GGA functionals

\begin{tabular}{|l|l|l|l|}
\hline ABINIT code & Exp. $\left(\mathbf{E}_{\mathbf{g}}(\mathbf{e V})\right)[\mathbf{8}]$ & $\left(\mathbf{E}_{\mathbf{g}}(\mathbf{e V})\right)$ & Functional \\
\hline ixc $=-201$ & & & \\
ixc $=-202$ & 0.335 & 2.080 & LTA \\
ixc $=-203$ & 0.335 & 0.390 & TPSS \\
ixc $=-204$ & 0.335 & 0.680 & M06L \\
ixc $=-205$ & 0.335 & 0.080 & GVT4 \\
ixc $=-206$ & 0.335 & 0.120 & TAU_HCTH \\
ixc $=-207$ & 0.335 & 0.110 & BR89 \\
ixc $=-208$ & 0.335 & 0.339 & BJ06 \\
ixc $=-209$ & 0.335 & 0.420 & TB09 \\
ixc $=-231$ & 0.335 & 0.400 & RPP09 \\
ixc $=-232$ & 0.335 & 0.000 & TPSS \\
\hline
\end{tabular}




\section{$\mathrm{FeS} 2$}

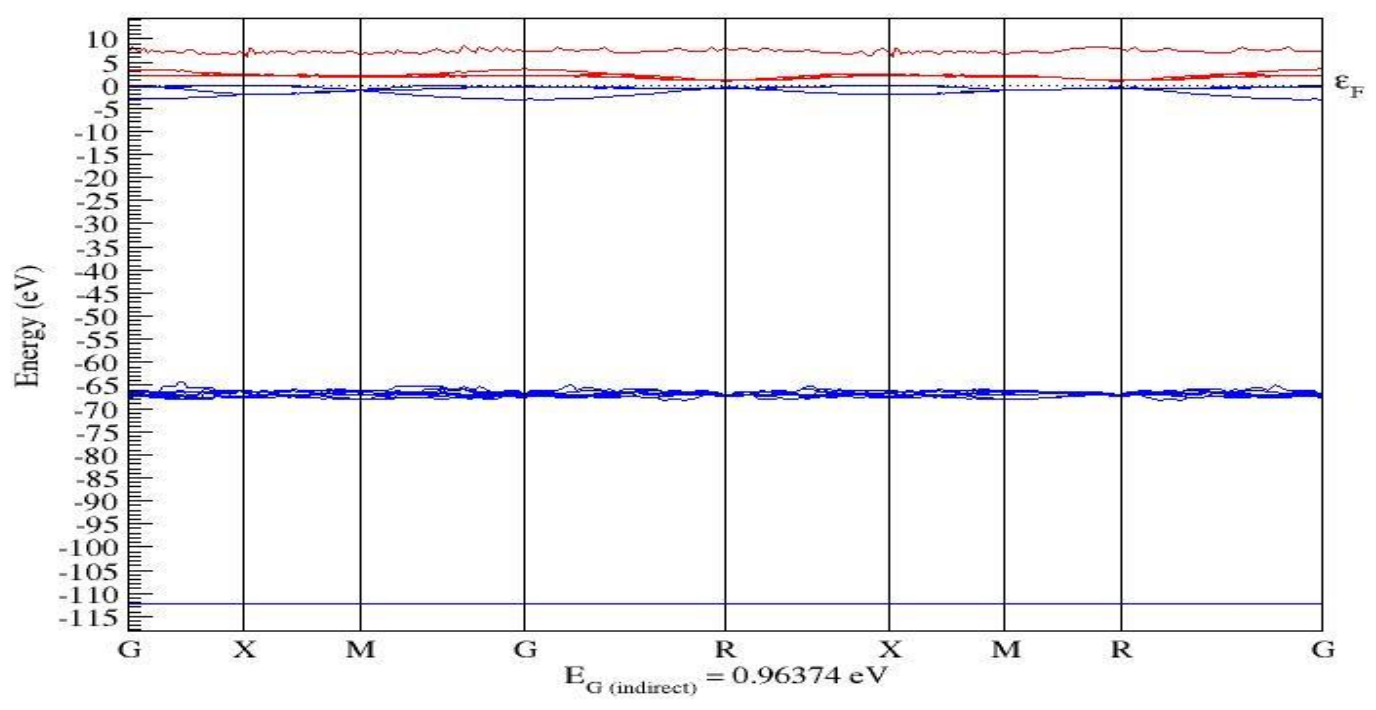

Figure 2: The Band Structure of Iron (II) disulfide using the M06L Functional

$\mathrm{Te}$

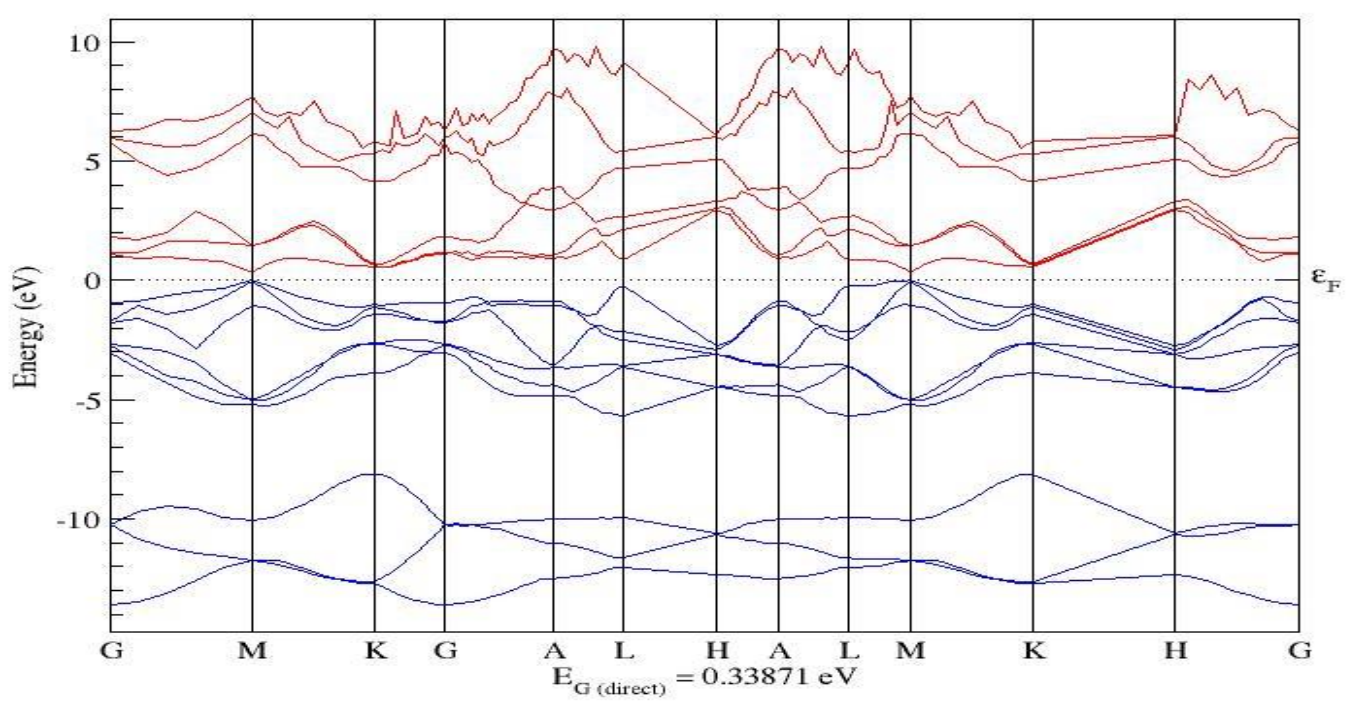

Figure 3: The Band Structure of Tellurium using the BJ06 Functional

For Tellurium the number of bands 15,20, 25 and 30 predicted it to be a semiconductor with different band gap values. The convergence of the band gap values by the meta gga functionals were achieved at $120 \mathrm{k}$-points for all the number of bands used except for the meta gga functionals (ixc $=-203$ and -232 ) which were achieved at $180 \mathrm{k}$-points. The meta gga functional (ixc =-207) gave band gap value for Tellurium that is closest to the experimental value. The results of the computed band gap of Iron (II) disulfide and Tellurium with the functionals are given in Tables 1 and 2. Figures 2 and 3 are the band structures of Iron (II) disulfide and Tellurium computed from the meta-GGA functionals (ixc $=-203$ and -207 ). 


\section{Conclusion}

In this paper, the band gap of Iron (II) disulfide and Tellurium were computed using the density functional theory with different meta-GGA functionals. The meta-GGA functionals M06L and BJ06 gave values of the band gap of Iron (II) disulfide and Tellurium closest to their experimental values. The meta-GGA functionals M06L and BJ06 can effectively predict the band gap of Iron (II) disulfide and Tellurium. In general, the use of meta-GGA functionals can be extended to compute the band gap of other semiconductors.

\section{How to Cite this Article:}

A. E. Arumona and A. N. Amah, "Density Functional Theory Calculation of Band Gap of Iron (II) Disulfide and Tellurium", Advanced Journal of Graduate Research, vol. 3, no. 1, pp. 41-46, Nov. 2017. doi: https://doi.org/10.21467/ajgr.3.1.41-46

\section{References}

[1] A. Schlegel, and P.Watchter, "Optical Properties, Phonons and Electronic Structure of Iron Pyrite", Environmental Science and Technology, vol. 42, no.6, pp.2168-2174, 1976.

[2] V. M. Fthenakis, K.C. Hyung, E. Alsema, "Emissions from Photovoltaic Life Cycles", Environmental Science and Technology, vol. 42 no.6, pp.2168-2174, 2008.

[3] K.Zweibel, ” The Impact of Tellurium Supply on Cadmium Telluride Photovoltaics”, Science, vol.328 no.5979, pp. 699-701, 2010.

[4] Y. Zhao, and D.G. Truhlar, "Calculation of Semiconductor Band Gaps with the M06-L Density Functional", The Journal of Chemical Physics, vol. 130, no.074103, pp. 1-3, 2009.

[5] Vijay et al., "Phase Stability and Thermoelectric Properties of the Mineral FeS 2 An Ab-inito Study", 2013.

[6] Qiu et al., "Density Functional Theory Calculation of Equilibrium Geometry and Electronic Structure of Pyrite", Trans. Nonferrous Met. Soc. China, vol. 11, no.4, pp.583-586, 2001.

[7] Y.N. Zhang, J. Hu, and R.Q.Wu, "Density Functional Theory (DFT) Studies for Iron Pyrite", 2011.

[8] H.G. Junginger, "Electronic Band Structure of Tellurium", Solid State Communications, vol.5, no.7, pp. 509-511, 1967, doi: 10.1016/00381098(67)90534-0

[9] C.J. Cramer, "Essentials of Computational Chemistry", $2^{\text {nd }}$ Ed. John Wiley \& Sons, 2004.

[10] Gonze, X., et al., "ABINIT : First-Principles Approach to Material and Nanosystem Properties", Computer Physics Communications, vol. 180, no.12, pp. 2582-2615, 2009.

[11] R.M. Martins, "Electronic Structure: Basic Theory and Practical Methods", Cambridge University Press, 2004.

[12] T. Bjorkman, "CIF2CELL: Generating Geometries for Electronic Structure Programs", Computer Physics Communications, vol. 182, no.5, pp.1183-1186, 2011.

[13] G. Brostigen, and A. Kjekshus,” Redetermine Crystal Structure of $\mathrm{FeS}_{2}$ (Pyrite)”, Acta Chemica Scandinavica, vol. 23, no.1, pp.21862188, 1969.

[14] Bradley, A.J. The Crystal Structures of the Rhombohedral Forms of Selenium and Tellurium. Philosophical Magazine, vol. 48, no.6, pp. 477-496, 1924.

[15] J.Tao, J.P.Perdew, V.N.Staroverov, and G.E. Scuseria, "Climbing the Density Functional Ladder: Nonempirical Meta-Generalized Gradient Approximation Designed for Molecules and Solids", Physical Review Letters, vol. 91, no.14, pp. 146401, 2003.

[16] Z. H.Yang, et al.," More Realistic Band Gaps from Meta-Generalized Gradient Approximation: Only In A Generalized Kohn-Sham Scheme," Phys. Rev. B 93, 205205, 2016.

[17] M.Ernzerhof, and G.E. Scuseria, "Kinetic Energy Density Dependent Approximations to the Exchange Energy", The Journal of Chemical Physics, vol. 111, no.3, pp. 911-916, 1999.

[18] Y.Zhao, and D.G. Truhlar, "A New Local Density Functional for Main-Group Thermochemistry, Transition Metal Bonding, Thermochemical Kinetics, and Noncovalent Interactions", The Journal of Chemical Physics, vol. 125, no.91, pp. 194101, 2006.

[19] V. T. Voorhis, and G.E. Scuseria, "A Novel Form for the Exchange-Correlation Energy Functional", Journal of Chemical Physics, vol. 109, no.2, pp. 400-410, 1998

[20] A. D. Boese, and N.C. Handy, "New Exchange-Correlation Density Functionals: The Role of the Kinetic-Energy Density", Journal of Chemical Physics, vol. 116, no.22, pp. 9559-9570. 2002.

[21] A. D.Becke, and M. R. Roussel," Exchange Holes in Inhomogeneous Systems: A Coordinate-Space Model”, Physical Review A, vol. 39, no.8, pp. 3761-3768, 1989.

[22] A. D.Becke, and E.R. Johnson, “A Simple Effective Potential for Exchange”, Journal of Chemical Physics, vol. 124, no.22, pp. 2211101, 2006.

[23] F.Tran, and P. Blaha, "Accurate Band Gaps of Semiconductors and Insulators with a Semilocal Exchange-Correlation Potential", Physical Review Letters, vol. 102, no.22, pp. 226401, 2009.

[24] E. Räsänen, S.Pittalis, and C.R. Proetto, "Universal Correction for the Becke-Johnson Exchange Potential", Journal of Chemical Physics, vol. 132, no.4, pp. 044112, 2010.

Publish your research article in AIJR journals- (Submit your article at journals.aijr.in )

$\checkmark$ Online Submission and Tracking

$\checkmark \quad$ Peer Reviewed

$\checkmark \quad$ Rapid decision

$\checkmark \quad$ Immediate Publication after acceptance

$\checkmark \quad$ Open Access (Articles freely available online)

$\checkmark \quad$ Retain full copyright of your article. 